\title{
Accuracy of VRS ionospheric corrections during ionospheric disturbances
}

\author{
Marta Krukowska, Paweł Wielgosz, Anna Krypiak-Gregorczyk \\ University of Warmia and Mazury in Olsztyn, Oczapowskiego 2, 10-719 Olsztyn, Poland
}

\begin{abstract}
This research presents accuracy analysis of ionospheric corrections embedded in VRS data stream provided by ASG-EUPOS reference network. The analysis are provided for disturbed ionospheric conditions that occurred in November 2013. The research are based on processing VRS station data and comparing the results to processing of real station data located in the same place. In particular, double-differenced ionospheric delay residuals are evaluated and compared. As a reference, double-differenced ionospheric delay residuals under quiet ionospheric conditions were also calculated. The results show that the quality of the ionospheric information embedded in VRS data stream deteriorates during ionospheric disturbances, however, it is still sufficient to support precise GNSS positioning.
\end{abstract}

Keywords: GNSS, Virtual Reference Stations (VRS), ionosphere.

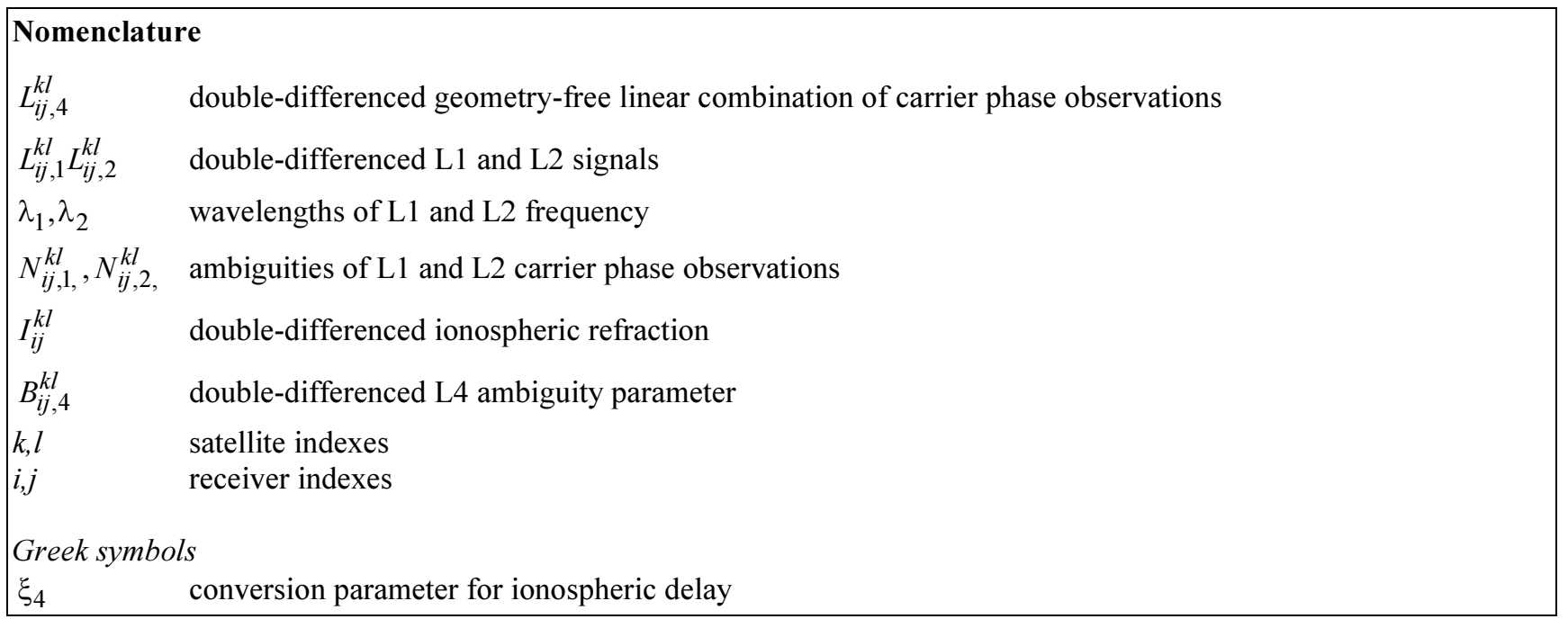

\section{Introduction}

Nowadays Virtual Reference Stations (VRS) corrections used in RTK (Real Time Kinematic) positioning have many well established applications in geodesy, architectural, road and railway engineering.

In 2008 Polish Head Office of Geodesy and Cartography established GBAS (Ground Base Augmentation System) in Poland named ASG-EUPOS. It is built according to European Position Determination (EUPOS) system standards. One of the tasks of ASG-EUPOS is provide users with data from reference stations. This data can be obtain in real-time or in post-processing mode. Users can choose whether they want to use data from the closes single-reference station or, over longer distances, correction stream [1-2].

Corresponding author: Marta Krukowska. E-mail address: marta.krukowska@uwm.edu.pl

http://dx.doi.org/10.3846/enviro.2014.225

(C) 2014 The Authors. Published by VGTU Press. This is an open-access article distributed under the terms of the Creative Commons Attribution License, which permits unrestricted use, distribution, and reproduction in any medium, provided the original author and source are credited. 
Hence there is a need to assess the accuracy of these corrections. This paper aims at accuracy analysis of the VRS ionospheric corrections provided by the ASG-EUPOS system.

\subsection{Ionosphere refraction and relative positioning}

The ionosphere is one of layers in the Earth atmosphere. It has significant influence on radio wave propagation and therefore on precise relative GNSS (Global Navigation Satellite Systems) positioning. The ionosphere delays pseudorange and advances carrier phase signals and also affects on ambiguity resolution. It is a dispersive medium with respect to GNSS signals - it has different influence for various frequencies and that property is used for estimation of its adverse effects on GNSS [3-6].

There are several methods to reduce this adverse influence. One of the methods is the usage of the Klobuchar model. It relies on ionospheric coefficients broadcast within GPS navigation message [7]. Another way to reduce ionospheric refraction is the usage of the NeQuick model. It uses monthly average values of solar activity either expressed by the 12-month running mean sunspot number or by the average $10.7 \mathrm{~cm}$ solar flux [6]. Next method is the application of global ionosphere maps provided by the International GNSS Service (IGS). The IGS ionospheric product is a combination of several independent solutions delivered by the IGS Analysis Centers in a form of global ionospheric maps [8]. This global model offers 2.5 by 5.0 degrees spatial and 2 hour temporal resolutions. Another frequently used possibility is the usage of ionosphere maps provided by Center for Orbit Determination in Europe (CODE). CODE model has similar temporal and spatial resolutions to the IGS product [9] and it is widely used in precise static positioning [10].

One of the most frequently used method to reduce the ionosphere delay is a relative positioning technique. The main concept of the relative positioning is to determine the coordinates of a unknown point with respect to a known point. Relative positioning requires simultaneous observations at both the reference and the unknown points, this allows forming double-differences (DD) of the original observations [6]. DDs cancel out receiver and satellite clock errors, and orbit errors and tropospheric and ionospheric delays are greatly reduced over short distances [11]. The Ionospheric delays can be neglected on baselines, up to $\sim 10 \mathrm{~km}$, depending on ionospheric conditions. However, over longer baselines the differential ionospheric residuals become larger and may hamper ambiguity resolution [12-13].

A popular method to reduce ionospheric influence over longer baselines is forming a ionosphere-free linear combination of the basic L1 and L2 GPS signals. This linear combination is often denoted as L3. It can be formed from both undifferenced and double-differenced data [9].

\subsection{VRS}

Although RTK positioning method has been widely in use since mid-90s, still important effort is put in order to enhance the performance of the precise relative positioning. This can be made by the application of the new GNSS signals [14-15] development of advanced methods for ambiguity resolution [16], as well as development new advanced methods for tropospheric and ionospheric refraction mitigation [17-18].

RTK method requires establishment of the reference stations. Nowadays many countries established national networks of permanent reference stations supporting precise GPS positioning, including RKT. Most of these networks are characterized by distances between the reference stations of about 70 kilometers. In effect the user can be away from the nearest reference station of about $35-50 \mathrm{~km}$. Over such distances the residual of orbital errors, tropospheric and ionospheric refractions are too great and classic RTK positioning cannot be carried out. In order to overcome these limitations a network-based RTK (RTN) concept was introduced. Three major RTN techniques are currently offered by the active reference networks: master and auxiliary concept (MAC) [19], Flachenkorrekturparameter (FKP) [20] or VRS [21]. Studies show that among those the VRS is the most popular.

The VRS data is obtained using the data from permanent reference stations network. The data from network is transferred to a network computing center, then ionospheric, tropospheric, clock and orbit errors are computed, next error models are used to predict the errors at the user location, VRS is created and virtual data is sent to the user [22]. This procedure, especially interpolation of errors causes some inaccuracy in VRS data in comparison to the data collected at the same place, but for real GPS receiver. Difference between this two cases can be treated as accuracy of VRS data. VRS to some extent solves problem of atmospheric delays decorrelation related with growing distance between reference station and user receiver [21].

However, it is expected that the VRS corrections may became inaccurate in case of the presence of atmospheric disturbances, the ionospheric storms in particular. Since, nowadays there are significant number of precise VRS applications, it is very important to examine VRS correction data accuracy.

\section{Experiment}

The goal of this research is to test the VRS correction accuracy during a geomagnetic storm. For that purpose the DD ionospheric delays obtained from the processing of real GPS data were compared to the DD ionospheric delays obtained from VRS data provided by the ASG-EUPOS system on the disturbed day of November $9^{\text {th }}, 2013$. 


\subsection{Methodology}

The GINPOS software developed at UWM (University of Warmia and Mazury in Olsztyn) was used to carry out the numerical tests [23]. Real user GPS data were collected (LEBO SmartNet station) and baselines to the closest ASG-EUPOS reference stations were formed. Then the baselines were processed and DD carrier phase ambiguities $N_{i j, 1,}^{k l}, N_{i j, 2}^{k l}$, were solved using the LAMBDA method [24]. Next, DD geometry-free linear combination (Eqn (1)) was formed using these real GPS data, and the "true" DD ionospheric delay was calculated (Eqn (3)). Mathematic model of the ionospheric delay calculation is given below.

$$
\begin{gathered}
L_{i j, 4}^{k l}=L_{i j, 1}^{k l}-L_{i j, 2}^{k l}=-\xi_{4} I_{i j}^{k l}+B_{i j, 4}^{k l} \\
B_{i j, 4}^{k l}=\lambda_{1} N_{i j, 1}^{k l}-\lambda_{2} N_{i j, 2}^{k l} \\
I_{i j}^{k l}=\frac{B_{i j, 4}^{k l}-L_{i j, 4}^{k l}}{\xi_{4}}
\end{gathered}
$$

where

$L_{i j 4}^{k l}$ is DD geometry-free linear combination of carrier phase observations,

$L_{i j 1}^{k l}, L_{i j 2}^{k l}$ are DD L1 and L2 phase observations,

$B_{i j, 4}^{k l}$ is an ambiguity parameter with an undefined wavelength, therefore expressed in units of length,

$\lambda_{1}, \lambda_{2}$ are wave lengths for L1 and L2 frequency,

$N_{i j, 1,}^{k l}, N_{i j, 2}^{k l}$, are ambiguities for L1 and L2 frequency,

$I_{i j}^{k l}$ is value of ionospheric refraction,

$\xi_{4}=1-\xi=1-\frac{v_{1}^{2}}{v_{2}^{2}} \approx-0.647$ is the factor that converts the ionospheric delay in $L_{4}$ to that of the first frequency,

$k, l$ are satellite indexes,

$i, j$ are receiver indexes.

In the next step VRS data were ordered at the exact location of the LEBO station for the same time period. Then, again, the same baselines connecting VRS station to neighboring ASG-EUPOS stations were formed. Next, DD ionospheric delays were calculated (Eqn (3)), this time based on VRS data.

The VRS ionospheric correction analysis is based on comparing these DD ionospheric delays derived from VRS data to the "real" DD ionospheric delays. The difference between ionospheric refraction for real station and VRS station - VRS residua - is the VRS ionospheric correction accuracy.

In should be noted that $\pm 0.05 \mathrm{~m}$ accuracy of the ionospheric corrections supports instantaneous positioning and $\pm 0.10 \mathrm{~m}$ accuracy is the limit for RTK (RTN) positioning [3]. Therefore correction residuals over $\pm 0.10 \mathrm{~m}$ mean that the VRS data is not accurate enough for the kinematic user requirements.

\subsection{Ionosphere conditions}

In order to test the VRS ionospheric correction accuracy during geomagnetic and ionospheric disturbances a geomagnetically active day of November $9^{\text {th }}, 2013$ was selected (DOY 313). The sum of the planetary $K p$ index amounted to $\Sigma K p=26+$ on that day. On the other hand, for a reference, a quiet day of November $25^{\text {th }}$ (DOY 329) with $\Sigma K p=0$ o was also selected. The average hourly ionospheric total electron content (TEC) values over the ASG-EUPOS network on the selected days are presented in Figure 1. In addition, Figure 2 presents the $K p$ index on the active day.

\subsection{Data source}

The test network was located in north part of Poland. Four ASG-EUPOS stations served as reference stations (REDZ, WLAD, GDAN, KOSC), and single LEBO station was selected as the test point (Fig. 3). Various GNSS receivers and antennas were used:

- TRM55971.00 TZGD antenna and Trimble NetR5 receiver at REDZ station,

- ASH701945E_M SNOW and Ashtech Micro Z (uZ-12) at WLAD,

- TRM41249.00 TZGD and Trimble NetRS at GDAN and KOSC,

- LEIAR25.R4 and LEICA GR10 serve as the user set at LEBO.

Four baselines were formed, their lengths ranged from $41 \mathrm{~km}$ to $62 \mathrm{~km}$ (Fig. 3). The VRS data was generated for exactly the same coordinates as LEBO station. It is very important that the test station belongs to different reference network 
system, so its data is not used for generation the VRS data. Dual-frequency GPS data with 30 seconds interval was processed in two 24 hour-long sessions.
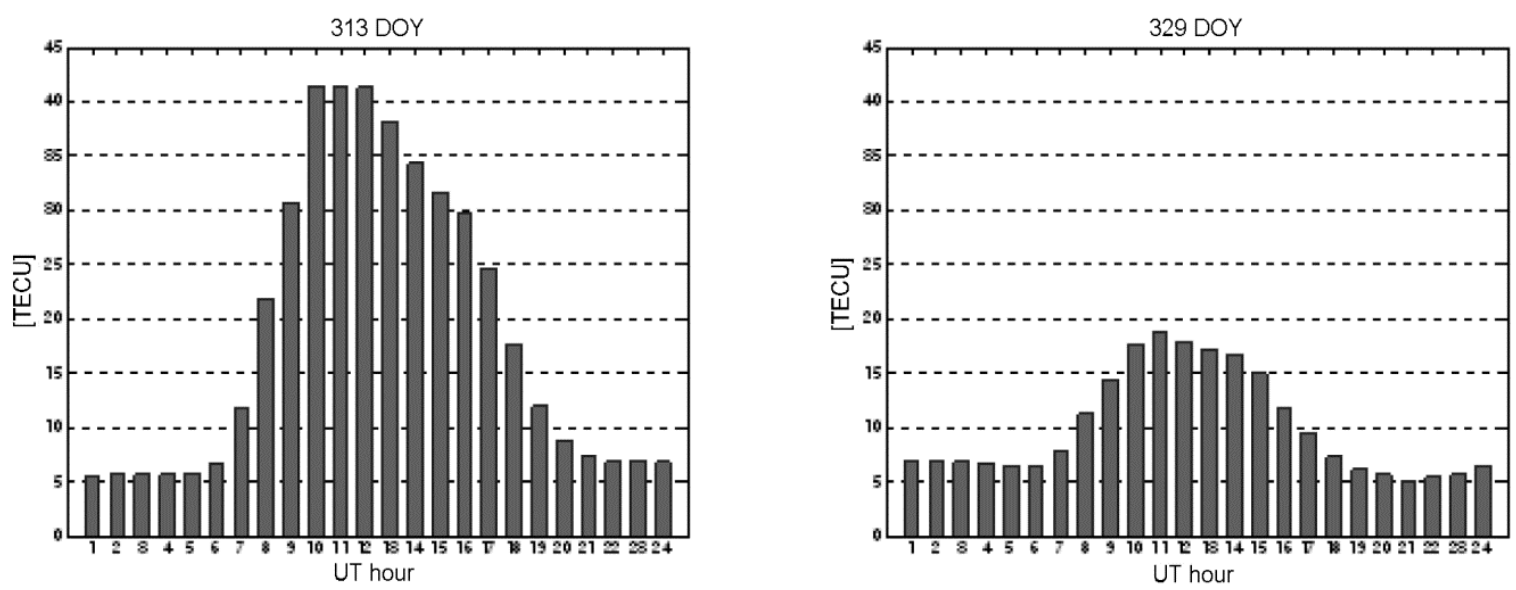

Fig. 1. Average hourly TEC over ASG-EUPOS network on DOYs 313 and 329/2013

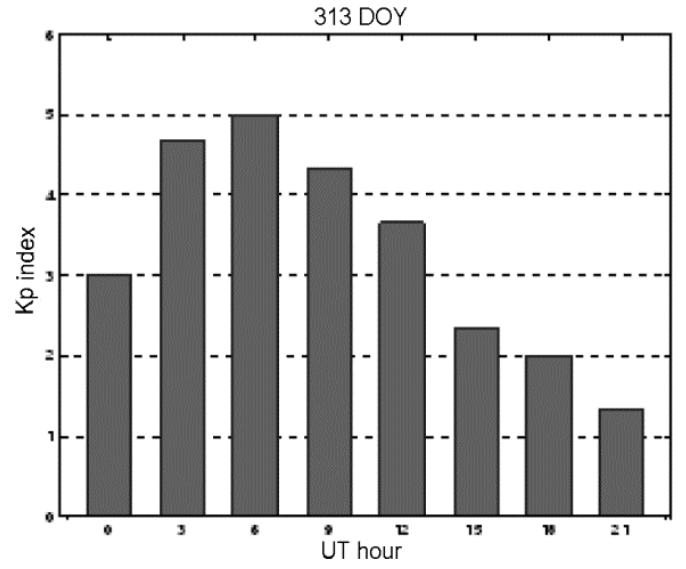

Fig. 2. Kp index on DOY 313

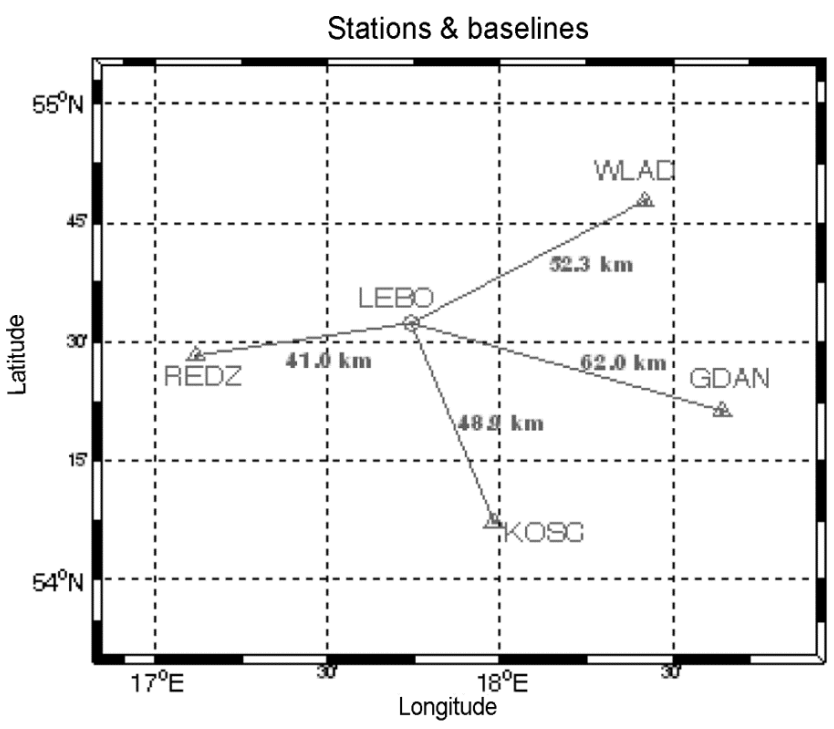

Fig. 3. The geometry of the test network and the tested baselines 


\section{Processing results}

Figure 4 shows "true" DD ionospheric delays obtained from actual GPS data for the quiet day (329 DOY). Most of ionosphere delays is smaller than $\pm 0.05 \mathrm{~m}$. At local noon (10:30 UT) there is maximum of ionospheric activity $(0.50 \mathrm{~m})$. Mean value of "true" DD ionospheric delays equals $0.042 \mathrm{~m}$ and their standard deviation is $0.058 \mathrm{~m}$. About $87 \%$ of ionospheric delays is smaller than $\pm 0.05 \mathrm{~m}, 96.3 \%$ is smaller than $\pm 0.10 \mathrm{~m}$ and less than $1 \%$ is larger than $\pm 0.20 \mathrm{~m}$ (Table 1 and 2). Figure 5 presents residuals of the VRS ionospheric corrections for the quiet day. It can be noticed that ionospheric delay is clearly reduced, $96.9 \%$ of the residuals are smaller than $\pm 0.05 \mathrm{~m}$ and there are no residuals exceeding $\pm 0.20 \mathrm{~m}$. Mean of residuals is reduced to $0.020 \mathrm{~m}$ and standard deviation is reduced to $0.027 \mathrm{~m}$ (Table 1 and 2).

Figure 6 shows "true" DD ionospheric delays obtained from actual GPS data for the disturbed day (313 DOY). During nighttime the ionospheric delay is smaller than $\pm 0.10 \mathrm{~m}$, but during daytime its values are more variable, and reach up to $\pm 0.60 \mathrm{~m}$. Mean delay reached $0.058 \mathrm{~m}$ and it is almost 1.5 times larger than mean for the quiet day. Almost $4 \%$ of ionospheric refraction is larger than $\pm 0.20 \mathrm{~m}$ (Table 1 and 2). Figure 7 shows the VRS ionospheric correction residuals for the disturbed day. The residuals are up to $\pm 0.40 \mathrm{~m}$ during daytime, which is already twice time more than L1 wavelength. On the other hand $98 \%$ of the residuals during 24 -hour period are below $\pm 0.10 \mathrm{~m}$ limit what may satisfy requirements of precise positioning.

More interesting results are obtained for daytime. We analyzed 8-hour time span between 6:00 UT and 14:00 UT (7:12-15:12 LT). It is time when the most of filed work is carried out. On the disturbed day during daytime only $64.8 \%$ of "true" DD ionospheric delay is smaller than $\pm 0.05 \mathrm{~m}$ and almost $10 \%$ is larger than $\pm 0.20 \mathrm{~m}$. At the same time only $85.50 \%$ of VRS ionospheric correction residuals are smaller than $\pm 0.05 \mathrm{~m}$. What is more important, more than $5 \%$ of the residuals are greater than $\pm 0.10 \mathrm{~m}$, what makes kinematic positioning impossible (Table 1 and 2).

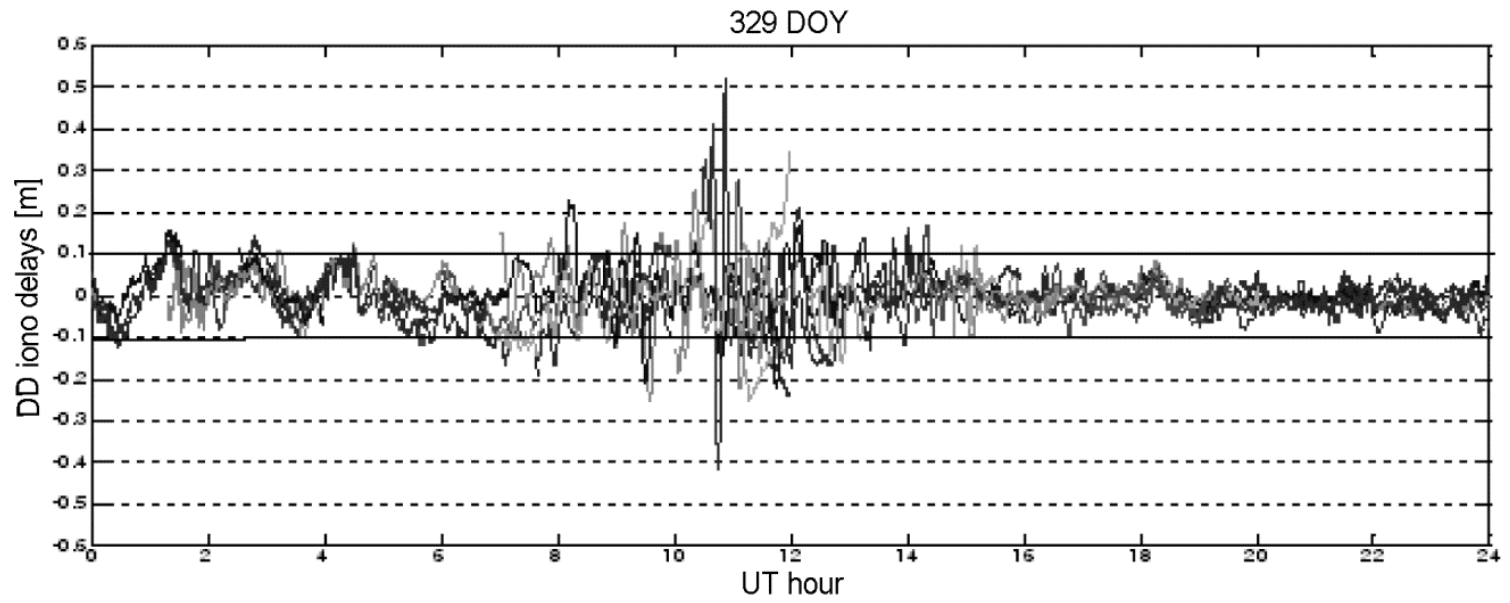

Fig. 4. "True" DD ionospheric delays on the quiet day (DOY 329)

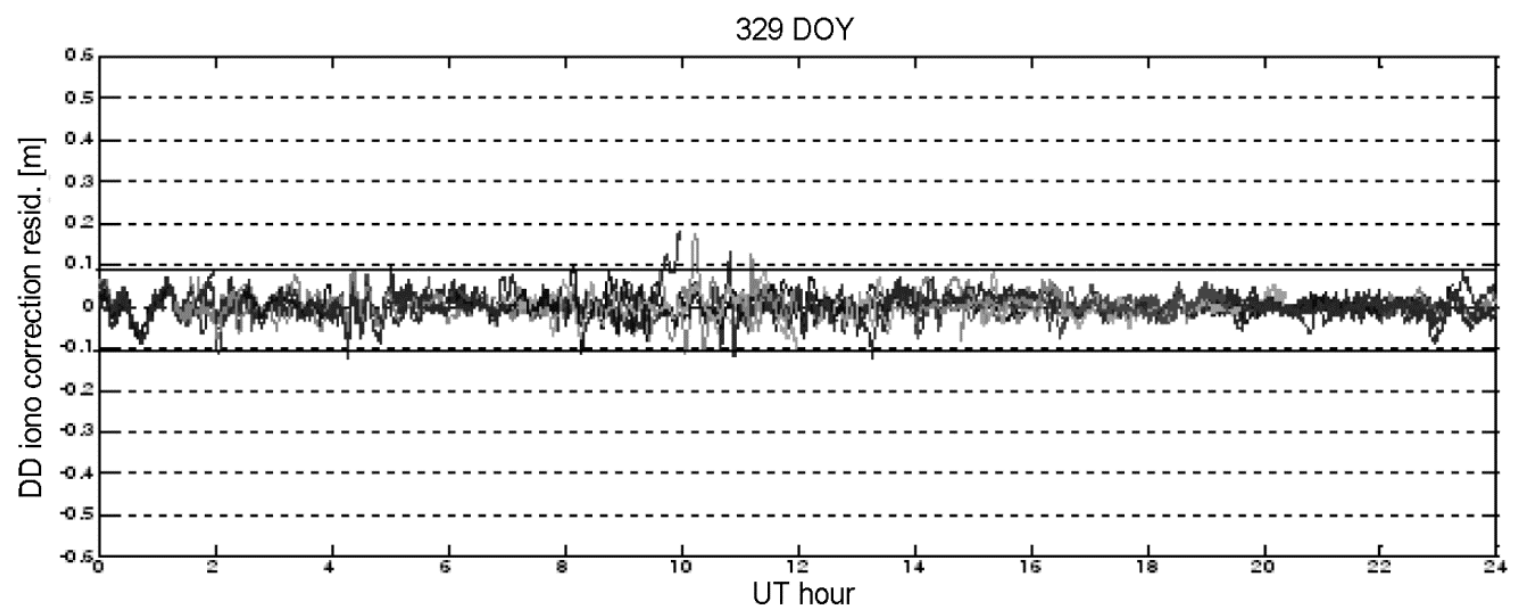

Fig. 5. VRS DD ionospheric correction residuals on the quiet day (DOY 329) 


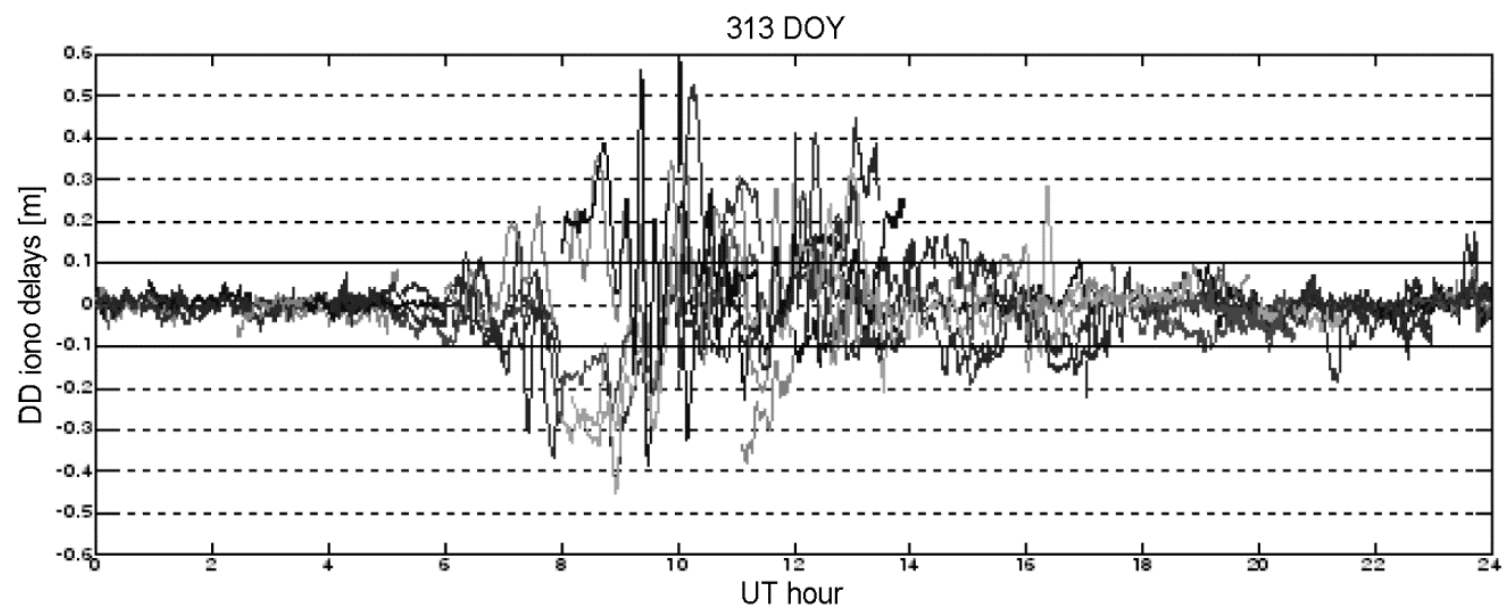

Fig. 6. "True" DD ionospheric delays on the disturbed day (DOY 313)

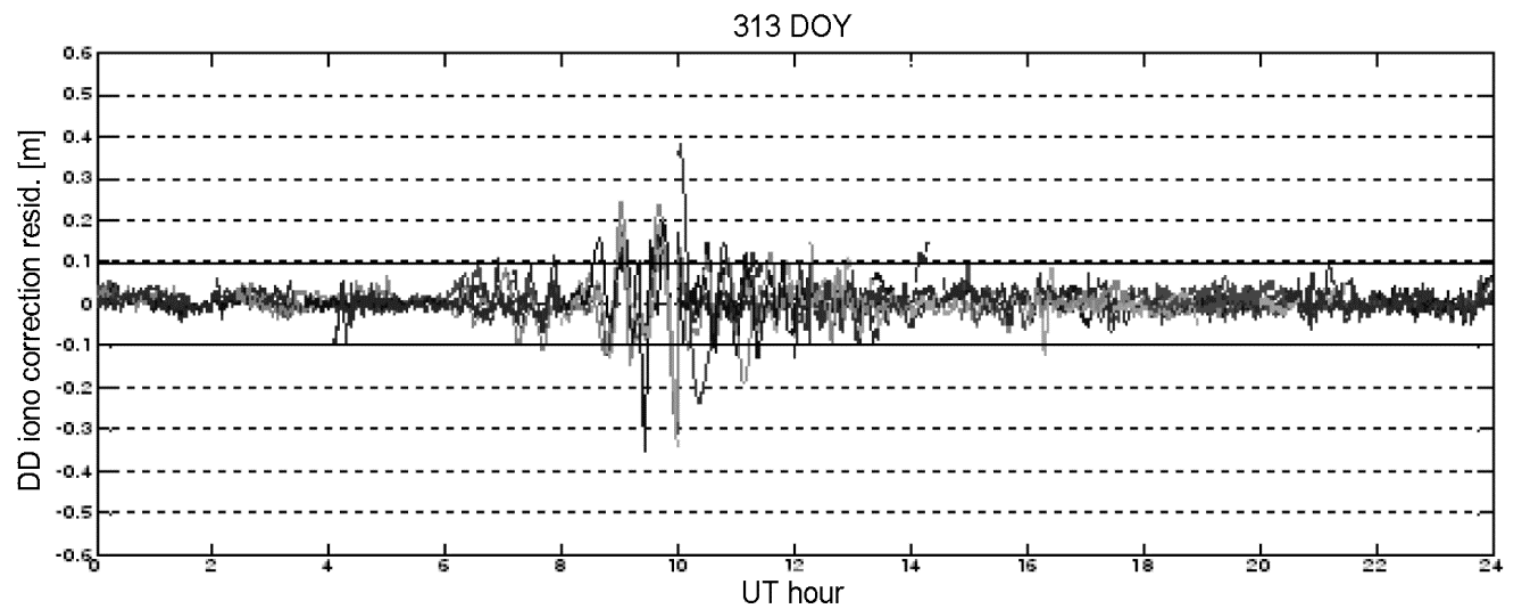

Fig. 7. VRS DD ionospheric correction residuals on the disturbed day (DOY 313)

Table 1. Mean and standard deviation of "true" DD ionospheric delay and VRS ionospheric correction residuals

\begin{tabular}{llllll}
\cline { 3 - 5 } & & \multicolumn{3}{l}{$8 \mathrm{~h}(6: 00-14: 00 \mathrm{UT})$} \\
\cline { 3 - 5 } $329 \mathrm{~h}(0: 00-24: 00 \mathrm{UT})$ & mean $[\mathrm{m}]$ & std $[\mathrm{m}]$ \\
\hline \multirow{2}{*}{329} & "true" & 0.042 & 0.058 & 0.062 & 0.082 \\
& VRS res. & 0.020 & 0.027 & 0.023 & 0.031 \\
\hline \multirow{2}{*}{313} & "true" & 0.058 & 0.094 & 0.114 & 0.150 \\
& VRS res. & 0.023 & 0.040 & 0.040 & 0.062 \\
\hline
\end{tabular}

Table 2. The percentage of the "true" DD ionospheric delays and VRS ionospheric correction residuals in predefined ranges

\begin{tabular}{clllllll} 
& & \multicolumn{2}{l}{$24 \mathrm{~h}(0: 00-24: 00 \mathrm{UT})$} & \multicolumn{3}{c}{$8 \mathrm{~h}(6: 00-14: 00 \mathrm{UT})$} \\
\cline { 3 - 8 } & & $\pm 0.05 \mathrm{~m}$ & $\pm 0.10 \mathrm{~m}$ & $\pm 0.20 \mathrm{~m}$ & $\pm 0.05 \mathrm{~m}$ & $\pm 0.10 \mathrm{~m}$ & $\pm 0.20 \mathrm{~m}$ \\
\hline \multirow{2}{*}{329} & "true" & $87.09 \%$ & $96.34 \%$ & $99.54 \%$ & $80.70 \%$ & $92.46 \%$ & $98.68 \%$ \\
& VRS res. & $96.92 \%$ & $99.77 \%$ & $100 \%$ & $95.44 \%$ & $99.34 \%$ & $100 \%$ \\
\hline \multirow{2}{*}{313} & "true" & $84.08 \%$ & $90.87 \%$ & $96.50 \%$ & $64.75 \%$ & $76.63 \%$ & $89.93 \%$ \\
& VRS res. & $94.01 \%$ & $98.06 \%$ & $99.79 \%$ & $85.50 \%$ & $94.74 \%$ & $99.40 \%$ \\
\hline
\end{tabular}

\section{Summary}

The ionospheric refraction has a significant influence on precise satellite positioning. Integer carrier phase ambiguities cannot be resolved in the presence of considerable un-modeled ionospheric delays. In order to limit this negative influence of the ionosphere networks of the reference stations are deployed. The reference networks provide the users ionospheric 
corrections supporting RTK (RTN) positioning. One of the methods for providing these corrections is VRS technique. This study analyses the accuracy of the VRS ionospheric corrections under different ionospheric conditions. It was shown that in general, the accuracy of the VRS corrections is sufficient. However, there are periods, especially during geomagnetic storms, when the accuracy of the correction deteriorates to the level when precise positioning cannot be supported.

\section{References}

[1] Bosy, J.; Graszka, W.; Leończyk, M. 2007. ASG-EUPOS. A Multifunctional Precise Satellite Positioning System in Poland, European Journal of Navigation 5(4): 2-6.

[2] Oruba, A.; Ryczywolski, M.; Wajda, S.; Bosy, J.; Graszka, W.; Leończyk, M.; Somla, J. 2008. The Implementation of the Precise Satellite Positioning System ASG-EUPOS, in ENC-GNSS 2008 Conference Proceedings, EUGIN, Toulouse (France).

[3] Wielgosz, P.; Kashani, I.; Grejner-Brzezińska, D. A. 2005. Analysis Of Long-Range Network RTK During Severe Ionospheric Storm, Journal of Geodesy 79(9): 524-531. http://dx.doi.org/10.1007/s00190-005-0003-y

[4] Wielgosz, P. 2011. Quality Assessment Of GPS Rapid Static Positioning With Weighted Ionospheric Parameters In Generalized Least Squares, GPS Solutions 15(2): 89-99. http://dx.doi.org/10.1007/s10291-010-0168-6

[5] Krypiak-Gregorczyk, A.; Wielgosz, P.; Gościewski, D.; Paziewski, J. 2013. Validation of Approximation Techniques For Local Total Electron Content Mapping, Acta Geodynamica et Geomaterialia 10(3): 275-283. http://dx.doi.org/10.13168/AGG.2013.0027

[6] Hofmann-Wellenhof, B.; Lichtenegger, H.; Wasle, E. 2008. GNSS-Global Navigation Satelite Systems. GPS, GLONASS, Galileo and more. Wien: SpringerWienNewYork. ISBN 978-3-211-73012-6

[7] Klobuchar, J. 1986. Design And Characteristics of the GPS Ionospheric Time-delay Algorithm For Single-frequency Users, PLANS'86 - Position Location and Navigation Symposium Conference Proceedings, Las Vegas, Nevada.

[8] Hernández-Pajares, M.; Juan, J. M.; Sanz, J.; Orus, R.; Garcia-Rigo, A.; Feltens, J.; Komjathy, A.; Schaer, S.; Krankowski, A. 2009. The IGS VTEC Maps: A Reliable Source Of Ionospheric Information Since 1998, Journal of Geodesy 83(3-4): 263-275. http://dx.doi.org/10.1007/s00190-008-0266-1

[9] Schaer, S. 1999. Mapping And Predicting The Earth's Ionosphere Using The Global Positioning System. Bern. PhD dissertation, Bern University

[10] Stepniak, K.; Baryła, R.; Wielgosz, P.; Kurpiński, G. 2013. Optimal data processing strategy in precise GPS leveling networks, Acta Geodynamica et Geomaterialia 10(172): 443-452. http://dx.doi.org/10.13168/AGG.2013.0044

[11] Sauer, K. 2003. Integrated High Precision Kinematic Positioning Using GPS and EGNOS Observations. London. PhD dissertation, University of London.

[12] Odijk, D. 2000 Weighting Ionospheric Corrections To Improve Fast GPS Positioning Over Medium Distances, in ION GPS 2000 Conference Proceedings, Salt Lake City, Utah.

[13] Kashani, I.; Wielgosz, P.; Grejner-Brzezinska, D. A. 2007. The Impact of the Ionospheric Correction Latency on Long-Baseline Instantaneous Kinematic GPS Positioning, Survey Review 39(305): 238-251. http://dx.doi.org/10.1179/175227007X197156

[14] Paziewski, J.; Wielgosz, P. 2013. Assessment of GPS + Galileo and multi-frequency Galileo single-epoch precise positioning with network corrections, GPS Solutions. Online-first. http://dx.doi.org/10.1007/s10291-013-0355- $\underline{3}$

[15] Teunissen, P. J. G.; Odolinski, R.; Odijk, D. 2013. Instantaneous BeiDou+GPS RTK positioning with high cut-off elevation angles, Journal of geodesy Online-first. DOI 10.1007/s00190-013-0686-4

[16] Cellmer, S.; Paziewski, J.; Wielgosz, P. 2013. Fast and precise positioning using MAFA method and new GPS and Galileo signals, Acta Geodynamica et Geomaterialia 10.4(172): 393-400.

[17] Wielgosz, P.; Paziewski, J.; Krankowski, K.; Kroszczyński, K.; Figurski, M. 2012. Results of the application of tropospheric corrections from different troposphere models for precise GPS rapid static positioning, Acta Geophysica 60(4): 1236-1257. http://dx.doi.org/10.2478/s11600-011-0078-1

[18] Wielgosz, P.; Krukowska, M.; Paziewski, J.; Krypiak-Gregorczyk, A.; Stepniak, K.; Kaplon, J.; Sierny, J.; Hadas, T.; Bosy, J. 2013. Performance of ZTD models derived in near real-time from GBAS and meteorological data in GPS fast-static positioning, Measurement Science and Technology 24: $125802(8 \mathrm{pp})$

[18] Brown, N.; Geisler, I.; Troyer, L. 2006. RTK Rover Performance Using The Master-Auxiliary Concept, Journal of Global Positioning Systems 5(1): 135-144. http://dx.doi.org/10.5081/jgps.5.1.135

[19] Wubbena, G.; Bagge, A. 2002. RTCM Message type 59-FKP for transmision of FKP, Geo++White paper 2002.01

[20] Vollath, U.; Buecherl, A.; Landau, H.; Pagels, Ch.; Wagner, B. 2000. Multi-Base RTK Positioning Using Virtual Reference Stations, ION GPS 2000 Conference Proceedings, Salt Lake City, Utah

[21] Vollath, U.; Buecherl, A.; Landau, H.; Pagels, Ch.; Wagner, B. 2000. Long-Range RTK Positioning Using Virtual Reference Stations, ION GPS 2000 Conference Proceedings, Salt Lake City, Utah

[22] Paziewski, J.; Wielgosz, P.; Krukowska, M. 2013. Application of SBAS pseudorange and carries phase signals to precise instantaneous singlefrequency positioning, Acta Geodynamica et Geomaterialia 10(172): 421-430. http://dx.doi.org/10.13168/AGG.2013.0041

[23] Teunissen, P. J. G. 1995. The Least-Squares Ambiguity Decorrelation Adjustment: A Method For Fast GPS Integer Ambiguity Estimation, Journal of Geodesy 70(1-2): 65-82. http://dx.doi.org/10.1007/BF00863419 